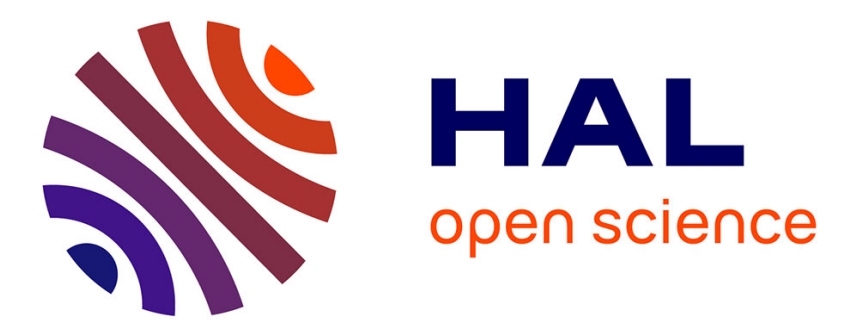

\title{
Use of a Virtual Environment to Engage Motor and Postural Abilities in Elderly Subjects With and Without Mild Cognitive Impairment (MAAMI Project)
}

Julien Bourrelier, Julien Ryard, Michèle Dion, Frédéric Merienne, Patrick Manckoundia, France Mourey

\section{To cite this version:}

Julien Bourrelier, Julien Ryard, Michèle Dion, Frédéric Merienne, Patrick Manckoundia, et al.. Use of a Virtual Environment to Engage Motor and Postural Abilities in Elderly Subjects With and Without Mild Cognitive Impairment (MAAMI Project). Innovation and Research in BioMedical engineering, 2016, 37 (2), pp.75-80. 10.1016/j.irbm.2016.02.007 . hal-01363542

\section{HAL Id: hal-01363542 \\ https://hal.science/hal-01363542}

Submitted on 9 Sep 2016

HAL is a multi-disciplinary open access archive for the deposit and dissemination of scientific research documents, whether they are published or not. The documents may come from teaching and research institutions in France or abroad, or from public or private research centers.
L'archive ouverte pluridisciplinaire HAL, est destinée au dépôt et à la diffusion de documents scientifiques de niveau recherche, publiés ou non, émanant des établissements d'enseignement et de recherche français ou étrangers, des laboratoires publics ou privés. 


\title{
Use of a Virtual Environment to Engage Motor and Postural Abilities in Elderly Subjects With and Without Mild Cognitive Impairment (MAAMI Project)
}

\author{
J. Bourrelier ${ }^{\mathrm{a}, *}$, J. Ryard $^{\mathrm{b}}$, M. Dion ${ }^{\mathrm{c}}$, F. Merienne ${ }^{\mathrm{b}}$, P. Manckoundia ${ }^{\mathrm{a}, \mathrm{d}}$, F. Mourey ${ }^{\mathrm{a}, \mathrm{e}}$ \\ a INSERM-U1093, UFR STAPS, Université Bourgogne Franche-Comté, Dijon, France \\ ${ }^{\mathrm{b}}$ CNRS Le2i Arts et Métiers ParisTech, Université Bourgogne Franche-Comté, Chalon-sur-Saône, France \\ ${ }^{\mathrm{c}}$ CNRS UMR 7366, Centre Georges Chevrier, Université Bourgogne Franche-Comté, UFR Sciences Humaines, Dijon, France \\ ${ }^{\mathrm{d}}$ Department of Geriatrics and Internal Medecine, University Hospital, Dijon, France \\ e INSERM-U1093, Université Bourgogne Franche-Comté, UFR Santé, Dijon, France
}

\begin{abstract}
Objectives: In the context of rehabilitation, the use of new technology such as Virtual Reality Technology (VRT) offers multiple possibilities to modulate the functional stimulation of subjects according to needs.

Material and methods: In this study, the validity and reliability of our VRT system were investigated in fifteen healthy aged adults (HAA) and seven aged subjects with mild cognitive impairment (MCI). One implicit session was designed through two virtual environments (VEs) in order to induce the adapted activities associated with balance and postural control. In comparison, the same activities were achieved in explicit sessions with a physiotherapist. This cross-over study made use of a qualitative method to collect participants' feelings in both implicit and explicit sessions.

Results: The results allowed us to validate the embedded technologies in our system and also the VE features for MCI subjects and their healthy counterparts. The analysis of participants' feelings showed that implicit tasks were preferred to explicit ones.

Conclusion: This study highlighted that the use of VRT generated a competitive spirit in all participants. This point underlines the major role of reward motivation in motor learning processes in rehabilitation.

(C) 2016 AGBM. Published by Elsevier Masson SAS. All rights reserved.
\end{abstract}

\section{Introduction}

In the context of rehabilitation and a prevention program linked to cognitive and motor impairment in persons with mild cognitive impairment (MCI) or in the early stage of Alzheimer's disease (AD), the use of new technology, such as Virtual Reality Technology (VRT) offers multiple possibilities to assess and stimulate functional abilities. The literature shows that this noninvasive and non-pharmacological approach is useful to assist

\footnotetext{
* Corresponding author at: INSERM U1093, Univ. Bourgogne FrancheComté, BP 27877, 21078 Dijon, France.

E-mail address: julien.bourrelier@u-bourgogne.fr (J. Bourrelier).
}

AD patients in cognitive activities of daily life [1,2]. Indeed, cognitive programs involve many types of exercises, which stimulate and/or assess spatial memory, executive functions and cognitive flexibility [3]. By contrast, the recent investigations highlighted the early increase in motor impairment in the course of the illness [4]. The main impairments reported in the literature concern gait and balance processes through anticipatory postural adjustment, which is affected in MCI and AD patients $[5,6]$.

VRT generates virtual environments (VEs) that provide the sensations associated with cognitive and motor tasks of daily life through realistic stimuli. Based on the degree of immersion and the level of interaction, these features play a crucial 
role in the representation of real-world situations $[7,8]$. For instance, fully-immersive VEs, which use 3D stereoscopic vision, provide a better representation of real-world situations and thus promote usual behavior during an assessment of patients' abilities [9]. However, this technical feature can induce cybersickness, a visual motion sickness, which can arise during or after immersion in VEs [10]. As regards the level of interaction, the reliability of VEs using an egocentric point of view (the player carries out the movement directly and not via an avatar) was studied in MCI subjects [11]. Finally, we have to determine the validity and reliability of VEs in aged people with cognitive impairment.

In rehabilitation or geriatric prevention programs the main difficulty is to take into account the characteristics and needs of individual aged people and to succeed in transferring the results to daily life. To this end, VEs offer various adapted activities in ecological conditions. Furthermore, the literature reports that the enrichment of a patient's environment is associated with the learning of new skills and an increase in brain reserves [12,13]. We also know that learning processes involve the stimulation of sensorimotor and cognitive abilities and also the activation of positive brain plasticity [14].

As regards motor learning, the literature also shows that implicit motor learning is better accepted and more reliable than explicit motor learning in $\mathrm{MCI}$ subjects and $\mathrm{AD}$ patients [15, 16]. The implicit learning of motor skills involves processes (a) which select and identify the environmental goals from sensorimotor interactions, (b) integrate a new relationship between the stimuli and the motor responses, (c) learn the sequencing of sub-movements related to the action required, and (d) translate the spatial targets into muscle commands [17]. Indeed, in implicit conditions, the desired movement emerges only from environment constraints and in the absence of specific instructions. Accordingly, the use of VEs seems appropriate to induce implicitly the realization of motor tasks by a subject with cognitive impairment.

In this preliminary study, the validity and reliability of our VRT system were investigated in MCI subjects and their healthy counterparts. To this end, two VEs were designed to induce the adapted activities associated with balance and postural control. This feasibility study made use of a qualitative method to collect participants' feelings.

\section{Materials and methods}

\subsection{Participants}

Twenty-two subjects aged over 70 years old participated in this experiment after giving written informed consent in accordance with the requirements of the local ethics committee (Dijon University Hospital, Dijon, France). Fifteen Healthy Aged Adults (HAA) made up the HAA group and seven participants with mild cognitive impairment made up the MCI group. The absence of dementia in the healthy volunteers was confirmed using standardized dementia tests and the mini mental state examination (MMSE) [18], which evaluates the cognitive capacities of subjects. The MCI participants followed a process
Table 1

Summary of subjects' demographic information.

\begin{tabular}{llll}
\hline & MCI $(n=7)$ & $\operatorname{HAA}(n=15)$ & $p$-value \\
\hline Age (years) & $79.1 \pm 4.7$ & $76.6 \pm 5.1$ & 0.28 \\
Gender Female (Male) & $4(3)$ & $6(9)$ & \\
MMSE $^{* *}$ Score & $23.2 \pm 2.4$ & $28.5 \pm 1.6$ & $<0.001$ \\
TUG (s) & $13.3 \pm 3.6$ & $9.5 \pm 2.1$ & 0.005 \\
\hline
\end{tabular}

** Indicates $p<0.001$ difference between groups. Data are presented as mean \pm SD. MMSE, Mini Mental State Examination; TUG, Timed Up and Go test.

of diagnosis based on NINCDS-ADRDA criteria, [19] which was created by a medical team in the Memory Center (Centre Mémoire de Ressources et de Recherche (CMRR) of Dijon University Hospital, Dijon, France). Participants were selected on the basis of the neuro-evaluations. In addition, inclusion was validated after obtaining consent from the participant. For the MCI group, the inclusion criterion was an MMSE score between 15 and 27. We also collected age and gender for each participant. Moreover, all subjects carried out the Timed Up and Go (TUG) test in order to determine their functional level [20] (see Table 1). All of the participants had normal vision and audition, whether corrected or not.

\subsection{Tasks and procedure}

\subsubsection{Description of VEs}

The VEs were designed using a visual immersion system called CAVE (Cave Automatic Virtual Environment). This environment is made up of two screens (resolution of 1024 by 768 pixels), the first was a front wall measuring $2.70 \mathrm{~m}$ high and $3.40 \mathrm{~m}$ wide and the second was a floor measuring $3 \mathrm{~m}$ depth and $3.40 \mathrm{~m}$ wide (see Fig. 1). First, the interactivity between the participant and the VE was supported by two complementary technologies. Indeed, this system uses active stereoscopic vision (NVidia 3D Vision Pro) with special 3D glasses. In addition, the system is designed in accordance with a tracking system composed of 4 infrared cameras (ART DTrack 2) operating at 60 frames per second with a precision of $1 \mathrm{~mm}$. Two parts of the participant's body are tracked, which allows an egocentric interaction in the VEs. Markers are positioned on the 3D glasses to capture movements of the participant's head. Other markers are placed on a wand, which is gripped by the participant to capture the movement of the dominant arm. The interaction between the participant and the VEs is managed using "iiVR" software.

\subsubsection{Implicit session in the VEs}

The implicit session included two "harvesting fruit" scenarios. They take place in an orchard atmosphere with a main tree in the center of the VE. In this VE, several targets are represented by several fruit (pears, apples, oranges). As regards the postural stimulation, we differentiated between two conditions for the action space: (1) in the peripersonal space (pps) (action without displacement of the feet) and (2) in the extrapersonal space (eps) (displacements are required) [21].

More precisely, in the implicit PPS condition (Ipps), the player maintains a standing position in front of the virtual wall. 


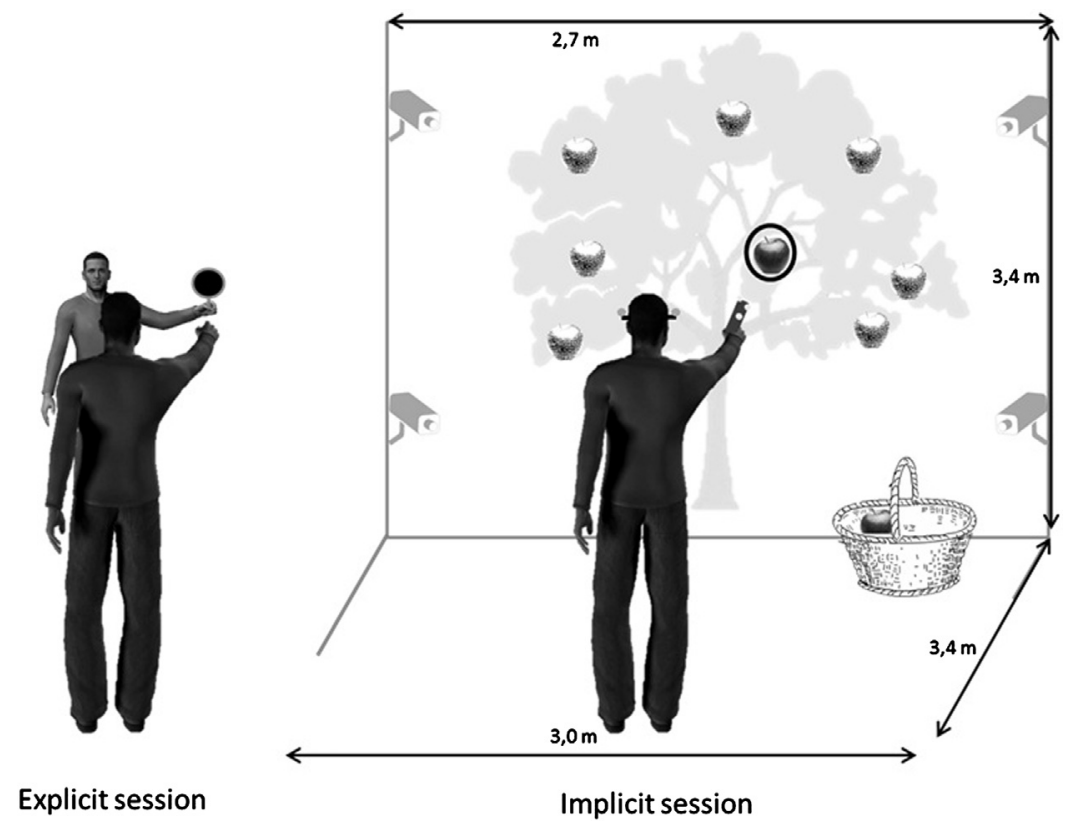

Fig. 1. Explicit therapy and implicit virtual environments.

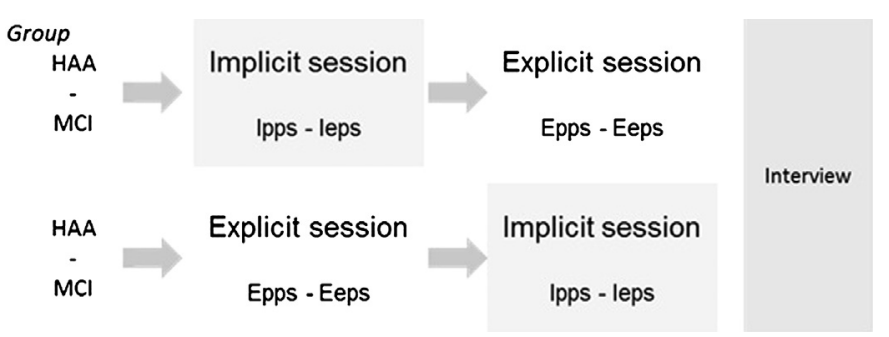

Fig. 2. Design of this cross-over study. HAA, healthy aged adults; MCI, Mild Cognitive Impairment; pps, peripersonal space condition and eps extrapersonal space condition in implicit and explicit session, I/E respectively.

He has to reach out as fast as possible with the dominant arm to pick a ripe fruit, which is defined before the start of the exercise. The ripeness is indicated by the color of the fruit, which goes through several stages (for instance, with an apple: from green to red and then to yellow). After picking the fruit, the player drops it in a virtual basket. The basket is located on the virtual floor between the front wall and the player. This spatial configuration implicitly restricts the displacement of the player. All of the fruit are displayed at the same location but the speed of color change varies randomly between 1 and $3 \mathrm{~s}$. The score is displayed at the end of exercise. The number of appropriate movements (nAM) corresponds to the number of ripe fruit harvested during the exercise.

The implicit EPS condition (Ieps) consists in harvesting a maximum of number of fruit and placing them in a virtual basket on the floor in $3 \mathrm{~min}$. The virtual fruit were located on the side of the player's dominant arm. Here, there were nine target positions. The nine fruit were displayed at the same time. Once the nine fruit had been harvested, nine new targets were displayed and so on. Simply, the score was the number of fruit harvested during the $3 \mathrm{~min}$. In this way, the VRT allows scenarios to be adapted to the abilities of subjects though the exercise features. For instance, we can change the time limit, the num- ber of trials, the number of targets, their positions on the front wall, the distance between them, their size, or even the fruits, their colors, and the position of basket... In this experiment, we adjusted the VE according to the length of the player's arm.

\subsubsection{Explicit session}

The explicit session was similar to a conventional physiotherapy session directed and managed by a physiotherapist. The therapist asked the subjects to perform an arm-pointing movement in two conditions. The same physiotherapist was used for all participants. The subject had to point explicitly to a target in the pps or eps spaces. The circular target was held by the physiotherapist. One side was "red" indicating preparation for the movement and the other side was "green" indicating the start of the movement. The physiotherapist used these visual stimuli to initiate movement. Moreover, he gave the instructions explicitly in order to obtain the desired movement according to both action spaces. The performance in these explicit conditions (Epps and Eeps) was also measured according to the nAM achieved by the subject.

\subsection{Design of the study}

The participants were distributed in the HAA and MCI groups. Each participant performed two sessions on the same day. Both the implicit session and explicit session comprised both types of exercise Ipps and Ieps, and Epps and Eeps, respectively. Here, a cross-over design was used to prevent a session order effect. Indeed, one half of each group was randomly selected to participate in the implicit session and then the explicit session, and vice versa for the other half (see Fig. 2). The implicit session included three Ipps exercises with nine targets to reach and three Ieps exercises with a time limit of $3 \mathrm{~min}$. There was a break of $1 \mathrm{~min}$ between each exercise repetition. The explicit session has the same features as the implicit con- 
ditions. The duration of both implicit and explicit sessions was approximatively $40 \mathrm{~min}$. In addition, we evaluated (1) the nAM performance of the subject during the implicit and explicit conditions and (2) the participants' feeling during and after the sessions through a qualitative method as described below.

\subsubsection{Interview to assess participants' feelings}

A qualitative method was used to collect participants' feelings about their personal experience during the experiment. All of the subjects participated in a semi-structured interview of $30 \mathrm{~min}$ in a quiet room. It was directed by a sociologist. Each interview was recorded on a smartphone in order to avoid stress in the subjects. Before the start of the experiment, the interview guidelines were validated in healthy subjects to assess their understanding and the reliability of sentence introduction and sentence stimulus given during the investigation. This investigation was divided into an observational stage and an interview stage in order to ensure that the behavior observed during the sessions was in accordance with the information provided in the interview. Specifically, this investigation focused on three main points: (1) the participants' relationship with new "technologies" including virtual reality, (2) their feelings about the interactivity, the comfort and the use of accessories in the VEs, and (3) their understanding of instructions and the game aspect of the implicit and explicit sessions.

\subsection{Data analysis}

The interview was conducted according to an interview grid, which allowed each interview to be analyzed according to the three main points. This grid provided the potential strengths and weaknesses of our system but also made it possible to compare implicit with explicit sessions.

The individual performance was obtained though the variable nAM, Beforehand, the homogeneity and normality of the variables were checked by the Levene test and the ShapiroWilk test, respectively. Specifically, Session (implicit and explicit) for two groups (HAA and MCI) was analyzed by means of repeated measures ANOVA. Post hoc analyses were conducted using HSD Tukey test. The alpha-level was set at $p=$ 0.05 .

\section{Results}

The participants in the both HAA and MCI groups did not differ in terms of age and education. The difference between the two groups for MMSE scores $(p<0.001)$ and the TUG test $(p=0.005)$ reflected cognitive impairment in MCI subjects, and an overall deficit in functional abilities compared with their healthy counterparts (see Table 1).

\subsection{Participants' feelings according to the interviews}

This investigation concerned all participants who had given oral consent for the recording and exploitation of their interviews. Three main points were developed through (1) the relationship with technologies, (2) the VEs and (3) the exercises.
First, the participants had varying degrees of experience with "new technologies". Most needed a computer to "conduct or handle business" but overall said they "did not play computer games". Some participants used computer technology "to communicate" with the family. Only a minority of the sample had not used a computer.

Interestingly, we noted that all participants reported personal experience with virtual environments in the cinema or in leisure parks but not in the context of gaming. Nonetheless, the use of a wand and 3D glasses did not surprise the participants, who accepted the requirement to use the wand to target the fruit. By contrast, some participants who needed glasses in everyday life were disturbed by the 3D glasses because "they slid down my nose, especially during downward movements". However, the participants reported no motion sickness during or after the implicit session.

For the second point, the participants enjoyed the game world through the orchard atmosphere and showed good perception of the elements in the set (fruit, tree, the basket, the score gauge, and the timer). However, several participants reported a lack of contrast between the green and yellow fruit and the tree in the PPS condition.

On the last point, the participants reported difficulty understanding the instructions in the explicit session. Specially, this point is confirmed in the stage of behavioral observation. Indeed, the physiotherapist gave a lot of instructions to adjust the movement parameters such as the foot position. However, the subjects said the explicit session, in comparison with the implicit session, was motivating and entertaining thanks to the presence of the physiotherapist. In addition, some participants reported a large number of repetitions specifically in the explicit session but not in the implicit session.

For all of the participants it was the first time they had played in a VE: "it was amusing, a game, a little tiring, not physically but in balance control". The observation stage confirmed that the participants were in a competitive spirit. Indeed, the majority of the subjects tried to improve their scores in different games. "I enjoyed it because I gathered 85 fruit in the first trial, 88 in the second and 89 in the third". This competitive spirit was slightly lower in MCI subjects.

\subsection{Performance in the implicit session}

As regards the results in the VE, a $2 \times 2$ ANOVA was conducted between Group (MCI, HAA) and Session (implicit and explicit). This analysis revealed a significant main effect for Session $(F(1.20)=30.70, p<0.001)$. The nAM was significantly greater in the implicit session than in the explicit session. In addition, this ANOVA showed a significant main effect for Group $(F(1.20)=16.47, p<0.001)$, which revealed a greater number of appropriate movements in healthy subjects than in MCI participants.

Interestingly, there was an interaction between the Session and the Group $(F(1.20)=6.67, p=0.01)$. The post-hoc analysis showed that MCI subjects carried out significantly fewer appropriate movements than their healthy counterparts in the implicit session $(p<0.001)$. In addition, this analysis revealed 


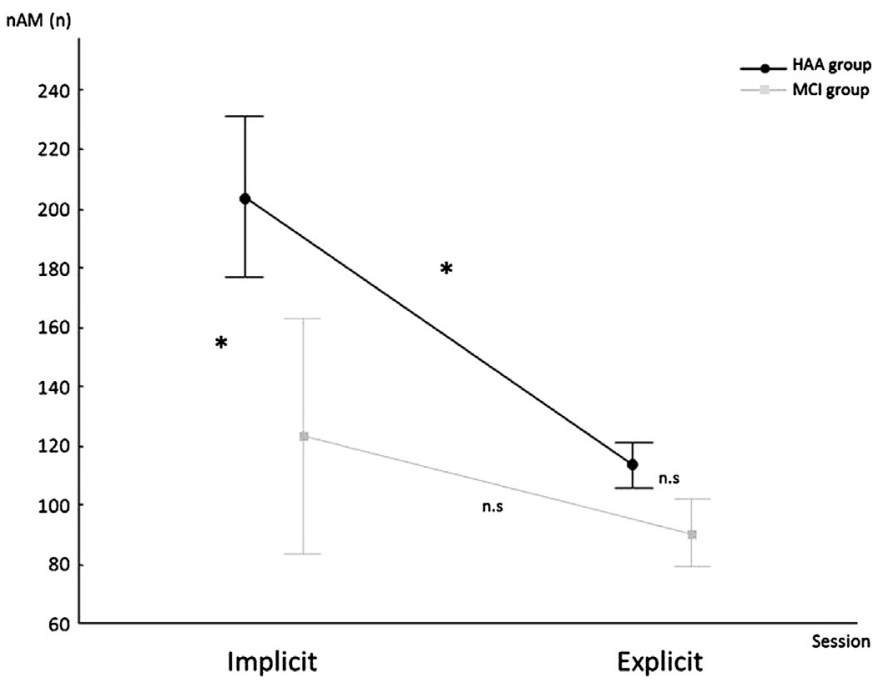

Fig. 3. nAM performance in sessions and groups. ${ }^{*}$ indicates $p \leq 0.001$ and n.s, not significant. nAM, number of appropriate movements.

that in the HAA group but not the MCI group the number of appropriate movements was significantly greater in the implicit session than in the explicit session $(p=0.001)$. Finally, there was no significant difference between the two groups for nAM in the explicit sessions (see Fig. 3).

\section{Discussion}

The aim of this preliminary study was to investigate the validity and reliability of our VRT system in aged subjects with and without cognitive impairment. In the context of motor training programs in MCI subjects and AD patients in the early stage of the disease, the literature advises the use of implicit situations in order to engage the motor abilities of users in suitable conditions [16]. In this way, we think that the use of VE makes it possible to adapt and enrich the subject's environment. This crucial point in rehabilitation promotes sensorimotor learning and cortical plasticity [22]. However, it seems essential to identify aged people's attitudes and feelings in order to design a tool that meets the needs of both the rehabilitation and the patient.

First, the qualitative investigation of participants' feelings allowed us to validate both the embedded technologies in our system and the VE features. The use of high level immersion with stereoscopic vision did not induce motion sickness during or after the implicit session in any of the novice participants. Indeed, the VEs were relatively stable. So, the orientation of the VE moves according to the movement of participant's head while the elements of VE were fixed in 3D space. As regards the VE features, all of the participants accepted the use of egocentric interactions. In our system, the subjects use the wand to harvest the fruit. Indeed, the literature suggests that the use of an egocentric point of view encourages embodiment in cognitive and motor tasks [23-25]. Moreover, the authors showed a greater deficit of information encoding and storage in memory tasks from an allocentric point of view (interactions between player and VEs are driven through an avatar) than from an egocentric point of view for MCI and AD patients especially [26].
In addition, this study compared two specific interventions with explicit and implicit sessions of motor and postural stimulation. The first aim was to highlight the acceptability and reliability of an implicit postural task compared with an explicit postural task often used in conventional therapy. Indeed, the literature shows that implicit methods improve the learning of motor tasks, especially among aged persons with cognitive impairment and in $\mathrm{AD}[27,28]$. Here, the analysis of participants' feelings confirmed the greater approval of implicit tasks than explicit ones. The participants revealed that the long instructions of the physiotherapist made it more difficult to understand the explicit conditions. Moreover, they also reported a feeling "of performing a large number of repetitions", which perhaps induced weariness during explicit sessions. In contrast, the implicit session was supported by a game and a new motivating factor for aged participants. Naturally, the use of new tool implicitly involves greater motivation and triggers curiosity. In these VEs, the performance score appeared as a reward and feedback. The literature widely highlights the major role of reward motivation in motor learning processes [29]. In this study, this involvement in the implicit session was confirmed in all participants through their desire to know their scores in the trials and their place in the ranking. In this way, performance analysis shows a greater number of appropriate movements in implicit sessions than in explicit sessions for an equal period of time.

In addition, this analysis of performance highlighted a significant difference between the HAA and MCI groups but only in the implicit sessions. We can think that the abilities of HAA subjects were underestimated by the type of assessment used in this study. Because of this, the physiotherapist may have imposed slower movements than the healthy participants were capable of. In contrast, movement frequency in VR was selfpaced by the subject. Moreover our results in MCI patients showed no significant differences between explicit and implicit sessions. We can think that our motor stimulation exercises caused greater difficulties in MCI patients than in healthy counterparts. These difficulties could be induced by the use of technology which may have disturbed the most vulnerable persons.

\section{Conclusion}

In this preliminary study, we confirmed the validity and reliability of our VRT system in subjects with or without cognitive impairment. In the next stage, we will try to demonstrate the validity of VEs in the rehabilitation context. Indeed, our aim is to use this system to stimulate and improve postural abilities in rehabilitation programs in patients with motor and/or cognitive impairments.

\section{Acknowledgements}

This work was supported by the French Research Agency (ANR) in Tecsan program (ANR-12-TECS-0014-01): MAAMI project (Maladie d'Alzheimer et Apprentissage Moteur Implicite - Alzheimer's disease and implicit motor learning). The authors had no conflict of interests. 


\section{References}

[1] Robert PH, König A, Amieva H, Andrieu S, Bremond F, Bullock R, et al. Recommendations for the use of serious games in people with Alzheimer's disease, related disorders and frailty. Front Aging Neurosci 2014;6:54. http://dx.doi.org/10.3389/fnagi.2014.00054.

[2] Jekel K, Damian M, Wattmo C, Hausner L, Bullock R, Connelly PJ, et al. Mild cognitive impairment and deficits in instrumental activities of daily living: a systematic review. Alzheimer's Res Ther 2015;7:17. http://dx.doi.org/10.1186/s13195-015-0099-0.

[3] García-Betances RI, Arredondo Waldmeyer MT, Fico G, CabreraUmpiérrez MF. A succinct overview of virtual reality technology use in Alzheimer's disease. Front Aging Neurosci 2015;7. http://dx.doi.org/10.3389/fnagi.2015.00080.

[4] Scarmeas N, Hadjigeorgiou GM, Papadimitriou A, Dubois B, Sarazin M, Brandt $J$, et al. Motor signs during the course of Alzheimer disease. Neurology 2004;63:975-82. http://dx.doi.org/10.1212/01.WNL.0000138440.39918.0C.

[5] Elble RJ, Leffler K. Pushing and pulling with the upper extremities while standing: the effects of mild Alzheimer dementia and Parkinson's disease. Mov Disord 2000;15:255-68.

[6] Tangen GG, Engedal K, Bergland A, Moger TA, Mengshoel AM. Relationships between balance and cognition in patients with subjective cognitive impairment, mild cognitive impairment, and Alzheimer disease. Phys Ther 2014;94:1123-34. http://dx.doi.org/10.2522/ptj.20130298.

[7] Costello P. Health and safety issues associated with virtual reality a review of current literature. Advis Gr Comput Graph Tech Rep Ser 1997. p. 1-23. http://citeseerx.ist.psu.edu/viewdoc/download?doi= 10.1.1.6.3025\&rep=rep $1 \&$ type $=$ pdf.

[8] Ma M, Zheng H. Virtual reality and serious games in healthcare. In: Advanced computational intelligence paradigms in healthcare 6. Virtual reality in psychotherapy, rehabilitation, and assessment. 2011. p. 169-92.

[9] Baus O, Bouchard S. Moving from virtual reality exposure-based therapy to augmented reality exposure-based therapy: a review. Front Human Neurosci 2014;8:1-15. http://dx.doi.org/10.3389/fnhum.2014.00112.

[10] Sharples S, Cobb S, Moody A, Wilson JR. Virtual reality induced symptoms and effects (VRISE): comparison of head mounted display (HMD), desktop and projection display systems. Displays 2008;29:58-69. http://dx.doi.org/10.1016/j.displa.2007.09.005.

[11] Weniger G, Ruhleder M, Lange C, Wolf S, Irle E. Egocentric and allocentric memory as assessed by virtual reality in individuals with amnestic mild cognitive impairment. Neuropsychologia 2011;49:518-27. http://dx.doi.org/10.1016/j.neuropsychologia.2010.12.031.

[12] Mirelman A, Maidan I, Herman T, Deutsch JE, Giladi N, Hausdorff JM. Virtual reality for gait training: can it induce motor learning to enhance complex walking and reduce fall risk in patients with Parkinson's disease?. J Gerontol, Ser A, Biol Sci Med Sci 2011;66:234-40. http://dx.doi.org/10.1093/gerona/glq201.

[13] Petrosini L, De Bartolo P, Foti F, Gelfo F, Cutuli D, Leggio $\mathrm{MG}$, et al. On whether the environmental enrichment may provide cognitive and brain reserves. Brains Res Rev 2009;61:221-39. http://dx.doi.org/10.1016/j.brainresrev.2009.07.002.
[14] Nithianantharajah J, Hannan AJ. Enriched environments, experiencedependent plasticity and disorders of the nervous system. Nat Rev Neurosci 2006;7:697-709. http://dx.doi.org/10.1038/nrn1970.

[15] Howard DV, Howard JH, Dennis N, LaVine S, Valentino K. Aging and implicit learning of an invariant association. J Gerontol, Ser B, Psychol Sci Soc Sci 2008;63:100-5.

[16] van Halteren-van Tilborg AD, Scherder EJ, Hulstijn W. Motorskill learning in Alzheimer's disease: a review with an eye to the clinical practice. Neuropsychol Rev 2007;17:203-12. http://dx.doi.org/10.1007/s11065-007-9030-1.

[17] Willingham DB. The neural basis of motor-skill learning. Curr Dir Psychol Sci 1999;8:178-82. http://dx.doi.org/10.1111/1467-8721.00042.

[18] Folstein MF, Folstein SE, McHugh PR. "Mini-mental state". A practical state method for grading the cognitive state of patients for the clinician. J Psychiatr Res 1975;12:189-98. http://dx.doi.org/10.1016/0022-3956(75)90026-6.

[19] Albert MS, DeKosky ST, Dickson D, Dubois B, Feldman HH, Fox NC, et al. The diagnosis of mild cognitive impairment due to Alzheimer's disease: recommendations from the National Institute on Aging-Alzheimer's Association workgroups on diagnostic guidelines for Alzheimer's disease. Alzheimer's Dement 2011;7:270-9. http://dx.doi.org/10.1016/j.jalz.2011.03.008.

[20] Podsiadlo D, Richardson S. The timed "Up \& Go": a test of basic functional mobility for frail elderly persons. J Am Geriatr Soc 1991;39:142-8.

[21] Rizzolatti G, Fadiga L, Fogassi L, Gallese V. The space around us. Science 1997;277:190-1.

[22] Kleim JA, Jones TA, Schallert T. Motor enrichment and the induction of plasticity before or after brain injury. Neurochem Res 2003;28:1757-69. http://dx.doi.org/10.1023/A:1026025408742.

[23] Grade S, Pesenti M, Edwards MG. Evidence for the embodiment of space perception: concurrent hand but not arm action moderates reachability and egocentric distance perception. Front Psychol 2015;6:1-9. http://dx.doi.org/10.3389/fpsyg.2015.00862.

[24] Serino S, Riva G. How different spatial representations interact in virtual environments: the role of mental frame syncing. Cogn Process 2015;16:191-201. http://dx.doi.org/10.1007/s10339-015-0646-4.

[25] Giummarra MJ, Gibson SJ, Georgiou-Karistianis N, Bradshaw JL. Mechanisms underlying embodiment, disembodiment and loss of embodiment. Neurosci Biobehav Rev 2008;32:143-60. http://dx.doi.org/10.1016/j.neubiorev.2007.07.001.

[26] Serino S, Morganti F, Di Stefano F, Riva G. Detecting early egocentric and allocentric impairments deficits in Alzheimer's disease: an experimental study with virtual reality. Front Aging Neurosci 2015;7:1-10. http://dx.doi.org/10.3389/fnagi.2015.00088.

[27] van Tilborg IADA, Hulstijn W. Implicit motor learning in patients with Parkinson's and Alzheimer's disease: differences in learning abilities? Mot Control 2010;14:344-61.

[28] Eldridge LL, Masterman D, Knowlton BJ. Intact implicit habit learning in Alzheimer's disease. Behav Neurosci 2002;116:722-6. http://dx.doi.org/10.1037/0735-7044.116.4.722.

[29] Wächter T, Lungu O, Liu T, Willingham D, Ashe J. Differential effect of reward and punishment on procedural learning. Brain Behav Immun 2009;29:436-43. http://dx.doi.org/10.1523/JNEUROSCI.4132-08.2009. 